\title{
Interim Estimates of Vaccine Effectiveness of Pfizer-BioNTech and Moderna COVID-19 Vaccines Among Health Care Personnel - 33 U.S. Sites, January-March 2021
}

Tamara Pilishvili, $\mathrm{PhD}^{1}$; Katherine E. Fleming-Dutra, $\mathrm{MD}^{1}$; Jennifer L. Farrar, $\mathrm{MPH}^{1}$; Ryan Gierke, $\mathrm{MPH}^{1}$; Nicholas M. Mohr, MD²; David A. Talan, MD 3 ; Anusha Krishnadasan, $\mathrm{PhD}^{3}$; Karisa K. Harland, $\mathrm{PhD}^{2}$; Howard A. Smithline, MD ${ }^{4}$; Peter C. Hou, MD ${ }^{5}$; Lilly C. Lee, MD ${ }^{6}$; Stephen C. Lim, MD 7 Gregory J. Moran, MD ${ }^{3}$; Elizabeth Krebs, MD ${ }^{8}$; Mark Steele, MD9; David G. Beiser, MD ${ }^{10}$; Brett Faine, PharmD 2; John P. Haran, MD, PhD ${ }^{11}$; Utsav Nandi, MD ${ }^{12}$; Walter A. Schrading, MD ${ }^{13}$; Brian Chinnock, MD ${ }^{14}$; Daniel J. Henning, MD ${ }^{15}$; Frank LoVecchio, DO ${ }^{16}$; Joelle Nadle, MPH ${ }^{17}$; Devra Barter, MSc ${ }^{18}$; Monica Brackney, MS ${ }^{19}$; Amber Britton, MPH ${ }^{20,21}$; Kaytlynn Marceaux-Galli, MPH ${ }^{22}$; Sarah Lim, MBBCh ${ }^{23}$; Erin C. Phipps, DVM ${ }^{24,25}$; Ghinwa Dumyati, MD ${ }^{26}$; Rebecca Pierce, PhD ${ }^{27}$; Tiffanie M. Markus, PhD ${ }^{28}$; Deverick J. Anderson, MD ${ }^{29}$; Amanda K. Debes, PhD ${ }^{30}$; Michael Lin, MD ${ }^{31}$; Jeanmarie Mayer, $\mathrm{MD}^{32}$; Hilary M. Babcock, MD 33 ; Nasia Safdar, MD, PhD ${ }^{34,35}$; Marc Fischer, MD ${ }^{1}$; Rosalyn Singleton, MD ${ }^{36}$; Nora Chea, MD ${ }^{1}$; Shelley S. Magill, MD, PhD ${ }^{1}$; Jennifer Verani, $\mathrm{MD}^{1}$; Stephanie Schrag, DPhil ${ }^{1}$; Vaccine Effectiveness Among Healthcare Personnel Study Team

On May 14, 2021, this report was posted as an MMWR Early Release on the MMWR website (https://www.cdc.gov/mmwr).

Throughout the COVID-19 pandemic, health care personnel (HCP) have been at high risk for exposure to SARS-CoV-2, the virus that causes COVID-19, through patient interactions and community exposure (1). The Advisory Committee on Immunization Practices recommended prioritization of HCP for COVID-19 vaccination to maintain provision of critical services and reduce spread of infection in health care settings (2). Early distribution of two mRNA COVID-19 vaccines (Pfizer-BioNTech and Moderna) to HCP allowed assessment of the effectiveness of these vaccines in a real-world setting. A test-negative case-control study is underway to evaluate mRNA COVID-19 vaccine effectiveness (VE) against symptomatic illness among HCP at 33 U.S. sites across 25 U.S. states. Interim analyses indicated that the VE of a single dose (measured 14 days after the first dose through 6 days after the second dose) was $82 \%$ (95\% confidence interval $[\mathrm{CI}]=74 \%-87 \%)$, adjusted for age, race/ethnicity, and underlying medical conditions. The adjusted VE of 2 doses (measured $\geq 7$ days after the second dose) was $94 \%(95 \% \mathrm{CI}=87 \%-97 \%)$. VE of partial (1-dose) and complete (2-dose) vaccination in this population is comparable to that reported from clinical trials and recent observational studies, supporting the effectiveness of mRNA COVID-19 vaccines against symptomatic disease in adults, with strong 2-dose protection.

A test-negative design case-control study of mRNA COVID-19 VE is underway, with HCP being enrolled at 33 sites across 25 U.S. states; the planned interim analysis presented in this report includes data collected during January-March 2021.* A majority (75\%) of enrolled HCP worked at acute care hospitals (including emergency departments), 25\% worked in outpatient or specialty clinics, and $<1 \%$ worked in long-term care facilities and urgent care

\footnotetext{
*https://www.cdc.gov/vaccines/covid-19/downloads/hcp-early-phaseprotocol-508.pdf
}

clinics. HCP with the potential for exposure to SARS-CoV-2 through direct patient contact or for indirect exposure (e.g., through infectious materials) were eligible for enrollment. ${ }^{\dagger}$ Case-patients and control participants (controls) were identified through routine employee testing performed based on site-specific occupational health practices. HCP with a positive SARS-CoV-2 polymerase chain reaction (PCR) or antigen-based test result and at least one COVID-19-like illness symptom ${ }^{\S}$ were enrolled as case-patients, and HCP with a negative SARS-CoV-2 PCR test result, regardless of symptoms, were eligible for enrollment as controls. Controls were frequency matched to case-patients (aiming for a ratio of three controls per case-patient) by site and week of test. HCP who reported having received a positive SARS-CoV-2 PCR or antigen-based test result $>60$ days earlier (i.e., with a previous SARS-CoV-2 infection) were excluded. Information on demographics, COVID-19-like illness symptoms within 14 days before or after the testing date, and presence of underlying conditions and risk factors for severe COVID-19 were collected through HCP interviews or self-completed surveys. Medical records were reviewed to collect data on SARS-CoV-2 test dates, type, and results and on medical care sought for COVID-19-like illness. Vaccination records, including dates and type of COVID-19 vaccine received, were obtained from occupational health or other verified sources (e.g., vaccine card, state registry, or medical record).

\footnotetext{
$\dagger$ https://www.cdc.gov/coronavirus/2019-ncov/hcp/guidance-risk-assesment-hcp.html ${ }^{\$}$ Health care personnel are considered symptomatic if one or more of the following signs and symptoms are present 14 days before or after the test date: fever (documented $\geq 100.4^{\circ} \mathrm{F}\left[38.0^{\circ} \mathrm{C}\right]$ or subjective), chills, cough (dry or productive), shortness of breath, chest pain or tightness, fatigue or malaise, sore throat, headache, runny nose, congestion, muscle aches, nausea or vomiting, diarrhea, abdominal pain, altered sense of smell or taste, loss of appetite, or red or bruised toes or feet.

$\checkmark$ Underlying conditions grouped based on CDC guidelines identifying conditions associated or potentially associated with risk for severe COVID-19 illness. https:/www.cdc.gov/coronavirus/2019-ncov/need-extra-precautions/peoplewith-medical-conditions.html
} 
HCP were defined as unvaccinated if they had not received any COVID-19 vaccine doses or had received their first dose after the test date. The interval of $0-13$ days from receipt of the first dose was defined as the time before first dose vaccine effect. The effectiveness of a single dose was measured during the interval from 14 days after the first dose through 6 days after the second dose. Because of the potential for vaccine-related reactions to influence HCP testing behaviors, sensitivity analyses of single-dose VE were conducted 1) excluding participants tested within $0-2$ days of receiving the second dose and 2) measuring $\mathrm{VE}$ before receiving the second dose. Effectiveness of 2 doses was measured $\geq 7$ days after the receipt of the second dose, consistent with the Pfizer-BioNTech clinical trial procedure (3). Sensitivity analyses measuring 2-dose effectiveness $\geq 14$ days after the second dose were conducted, consistent with the Moderna clinical trial procedure (4). Conditional logistic regression was used to estimate matched odds ratios (mORs) adjusted for age, race/ethnicity, and presence of underlying conditions. VE was estimated as $100 \% \times(1-\mathrm{mOR})$ for 1 or 2 doses, compared with no doses. Because of the small sample size, analyses could not be stratified by COVID-19 vaccine type. All statistical analyses were conducted using SAS (version 9.4; SAS Institute). This activity was reviewed by CDC and was conducted consistent with applicable federal law and CDC policy.**

As of March 18, 2021, 623 case-patients and 1,220 controls had been enrolled. The median ages of case-patients and controls were 38 years (range $=19-69$ years) and 37 years (range $=19-76$ years), respectively $($ Table 1$)$. The majority of HCP ( $60 \%$ of case-patients and $64 \%$ of controls) worked in occupational categories with substantial anticipated direct patient contact and were aged $19-49$ years $(75 \%$ and $76 \%$, respectively), female ( $84 \%$ and $82 \%$, respectively), and non-Hispanic White (64\% and 70\%, respectively). Underlying conditions associated with increased risk for severe COVID-19 were reported by $77 \%$ of case-patients and $75 \%$ of controls. Case-patients were significantly more likely than controls to have fever $(40 \%$ versus $23 \%, \mathrm{p}<0.001)$, cough $(56 \%$ versus $22 \%, \mathrm{p}<0.001)$, or shortness of breath $(26 \%$ versus $7 \%$, $\mathrm{p}<0.001) ; 5 \%$ of case-patients and $14 \%$ of controls reported only mild symptoms (sore throat, headache, runny nose, or congestion; $\mathrm{p}<0.001) ; 17 \%$ of controls reported no symptoms. Only $12(2 \%)$ case-patients and $10(1 \%)$ controls had severe illness requiring hospitalization, and no deaths occurred in either group.

Ten percent of case-patients and $20 \%$ of controls had received 1 dose of COVID-19 vaccine $\geq 14$ days before

\footnotetext{
** This investigation was defined as having met the requirements for public health surveillance as defined in 45 C.F.R. part 46.102(1)(2) 21 C.F.R. part 56; 42 U.S.C. Sect. 241(d); 5 U.S.C. Sect. 552a; 44 U.S.C. Sect. 3501 et seq.
}

the test date, and $3 \%$ of case-patients and $15 \%$ of controls had received 2 doses $\geq 7$ days before the test date (Table 2). Among vaccinated persons, $76 \%$ of case-patients and $78 \%$ of controls received the Pfizer-BioNTech vaccine; the remainder received the Moderna vaccine. The adjusted single-dose VE was $82 \%(95 \% \mathrm{CI}=74 \%-87 \%)$ and was similar for both 1-dose sensitivity analyses (before dose 2 : VE $=74 \%$, $95 \% \mathrm{CI}=62 \%-82 \%$; excluding days $0-2$ after dose 2 : $\mathrm{VE}=78 \%, 95 \% \mathrm{CI}=68 \%-84 \%)$. The adjusted 2-dose VE was $94 \%$ (95\% CI = 87\%-97\%); effectiveness $\geq 14$ days after the second dose was similar $(\mathrm{VE}=90 \%, 95 \% \mathrm{CI}=77 \%-96 \%)$.

\section{Discussion}

This multisite test-negative design case-control study found that authorized mRNA COVID-19 vaccines (Pfizer-BioNTech and Moderna) are highly effective against symptomatic COVID-19 among HCP. Effectiveness of a complete 2-dose regimen of these vaccines was estimated to be $94 \%$, consistent with findings from two clinical trials $(3,4)$. Although the case definition applied in this study was broader than that used in both clinical trials $(3,4), 93 \%$ and $88 \%$ of cases included in this study met the respective Pfizer-BioNTech and Moderna trial case definitions. The results are also consistent with findings from an observational study among the general adult population from Israel (5), two cohort studies among HCP from the United Kingdom, ${ }^{\dagger \dagger}$ and recently reported interim results from a U.S. cohort evaluation among HCP and frontline workers ( 6 ).

Effectiveness of a single dose, estimated to be $82 \%$ in this report, has also been demonstrated in phase III trials and recent observational studies. The estimated effectiveness found in this report is higher than estimates of single-dose effectiveness found in the Pfizer-BioNTech clinical trial (efficacy 52\%; $95 \% \mathrm{CI}=30 \%-68 \%$ ) (3) and an observational study from Israel (5). In the Israeli study, the Pfizer-BioNTech VE against symptomatic illness among the general adult population was $57 \%(95 \% \mathrm{CI}=50 \%-63 \%)$ and $66 \%(95 \% \mathrm{CI}=57 \%-73 \%)$ measured during 14-20 and 21-27 days, respectively, after the first dose (5). These differences might be related to the younger age of the HCP population in this study ( $<2 \%$ of participants aged $\geq 65$ years) compared with the age of the Israeli study population ( $13 \%$ aged $\geq 70$ years). In two cohort studies among HCP, the single-dose effectiveness of the Pfizer-BioNTech vaccine was consistent with the estimates in this report, with $72 \%$ effectiveness $(95 \% \mathrm{CI}=58 \%-86 \%) 21$ days after the first dose in a U.K. study (7) and $80 \%$ effectiveness $(95 \% \mathrm{CI}=59 \%-90 \%) \geq 14$ days after the first dose in a U.S. cohort study (の). Because the single-dose

\footnotetext{
†† https://doi.org/10.1101/2021.03.09.21253218; https://doi org/10.1101/2021.03.11.21253275
} 
TABLE 1. Characteristics of health care personnel case-patients and controls - 33 U.S. sites, January-March 2021

\begin{tabular}{|c|c|c|}
\hline \multirow[b]{2}{*}{ Characteristic } & \multicolumn{2}{|c|}{ No. (\%) } \\
\hline & $\begin{array}{l}\text { Case-patients* } \\
\qquad(\mathrm{N}=623)\end{array}$ & $\begin{array}{l}\text { Controls* } \\
(N=1,220)\end{array}$ \\
\hline \multicolumn{3}{|l|}{ Age group, yrs } \\
\hline Median (range) & $38(19-69)$ & $37(19-76)$ \\
\hline $19-49$ & $470(75)$ & $931(76)$ \\
\hline $50-64$ & $144(23)$ & $257(21)$ \\
\hline$\geq 65$ & $7(1)$ & $24(2)$ \\
\hline Missing & $2(<1)$ & $8(<1)$ \\
\hline \multicolumn{3}{|l|}{ Sex } \\
\hline Male & $99(16)$ & $223(18)$ \\
\hline Female & $521(84)$ & $996(82)$ \\
\hline Other & $3(<1)$ & $1(<1)$ \\
\hline \multicolumn{3}{|l|}{ Race/Ethnicity } \\
\hline White, non-Hispanic & $401(64)$ & $853(70)$ \\
\hline Black, non-Hispanic & $64(10)$ & $64(5)$ \\
\hline Hispanic/Latino & $81(13)$ & $124(10)$ \\
\hline Other ${ }^{\dagger}$ & $77(13)$ & $179(15)$ \\
\hline \multicolumn{3}{|l|}{ Anticipated level of HCP patient contact based on occupational category } \\
\hline Substantial ${ }^{\S}$ & $375(60)$ & $785(64)$ \\
\hline Moderate & $60(10)$ & $120(10)$ \\
\hline Minimal $^{* *}$ & $147(24)$ & $221(18)$ \\
\hline Undefined $^{+t}$ & $41(7)$ & $94(8)$ \\
\hline $\begin{array}{l}\text { Presence of one or more underlying conditions or risk factors associated with } \\
\text { increased risk for severe COVID-19\$§ }\end{array}$ & $480(77)$ & $920(75)$ \\
\hline Obesity (BMI $>30 \mathrm{~kg} / \mathrm{m}^{2}$ or listed in medical record) & $217(35)$ & $395(32)$ \\
\hline Overweight (BMI $25-29 \mathrm{~kg} / \mathrm{m}^{2}$ or listed in medical record) & $186(30)$ & $355(29)$ \\
\hline Asthma & $98(16)$ & $211(17)$ \\
\hline Hypertension & $92(15)$ & $159(13)$ \\
\hline 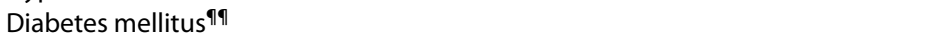 & $28(4)$ & $57(5)$ \\
\hline Immunocompromising condition ${ }^{* * *}$ & $25(4)$ & $46(4)$ \\
\hline Heart disease & $15(2)$ & $61(5)$ \\
\hline Cerebrovascular disease & $2(<1)$ & $4(<1)$ \\
\hline Neurologic condition & $2(<1)$ & $7(<1)$ \\
\hline Chronic kidney disease & $1(<1)$ & $5(<1)$ \\
\hline Chronic obstructive pulmonary disease & $1(<1)$ & $6(<1)$ \\
\hline Other chronic lung disease & $6(<1)$ & $16(1)$ \\
\hline Chronic liver disease & $2(<1)$ & $6(<1)$ \\
\hline Current or former smoking ${ }^{t+\dagger}$ & $130(21)$ & $255(21)$ \\
\hline Pregnancy (proportion among female HCP) & $13(3)$ & $40(4)$ \\
\hline \multicolumn{3}{|l|}{ Reported symptoms of illness } \\
\hline Fever (measured temperature $\geq 100.4^{\circ} \mathrm{F}\left[38.0^{\circ} \mathrm{C}\right]$ or subjective) ${ }^{\S \S \S}$ & $249(40)$ & $281(23)$ \\
\hline Cough (dry or productive) $\S_{\S \S}$ & $348(56)$ & $267(22)$ \\
\hline Shortness of breath $\S \S \S$ & $161(26)$ & $80(7)$ \\
\hline Chills $\S^{\S}$ & $275(44)$ & $324(27)$ \\
\hline Muscle pain $\S \S \S$ & $289(46)$ & $342(28)$ \\
\hline Altered sense of smell or taste $\mathrm{e}^{\S \S}$ & $351(56)$ & $45(4)$ \\
\hline Sore throat ${ }^{\S \S \S}$ & $215(35)$ & $344(28)$ \\
\hline Diarrhea ${ }^{\S \S}$ & $154(25)$ & $173(14)$ \\
\hline Nausea or vomiting $§ \S \S$ & $132(21)$ & $186(15)$ \\
\hline Other symptoms sศๆๆ & $560(90)$ & $796(65)$ \\
\hline Hospitalized & $12(2)$ & $10(1)$ \\
\hline \multicolumn{3}{|l|}{ COVID-19 vaccine status } \\
\hline Unvaccinated & $340(55)$ & $302(25)$ \\
\hline Received $\geq 1$ dose before test date, by vaccine type & $283(45)$ & $918(75)$ \\
\hline Pfizer-BioNTech & $214(76)$ & $712(78)$ \\
\hline Moderna & $68(24)$ & $200(22)$ \\
\hline Mixed product ${ }^{* * * *}$ & 0 & $1(0.4)$ \\
\hline Missing product information & $1(0.4)$ & $5(0.5)$ \\
\hline
\end{tabular}

See table footnotes on the next page. 


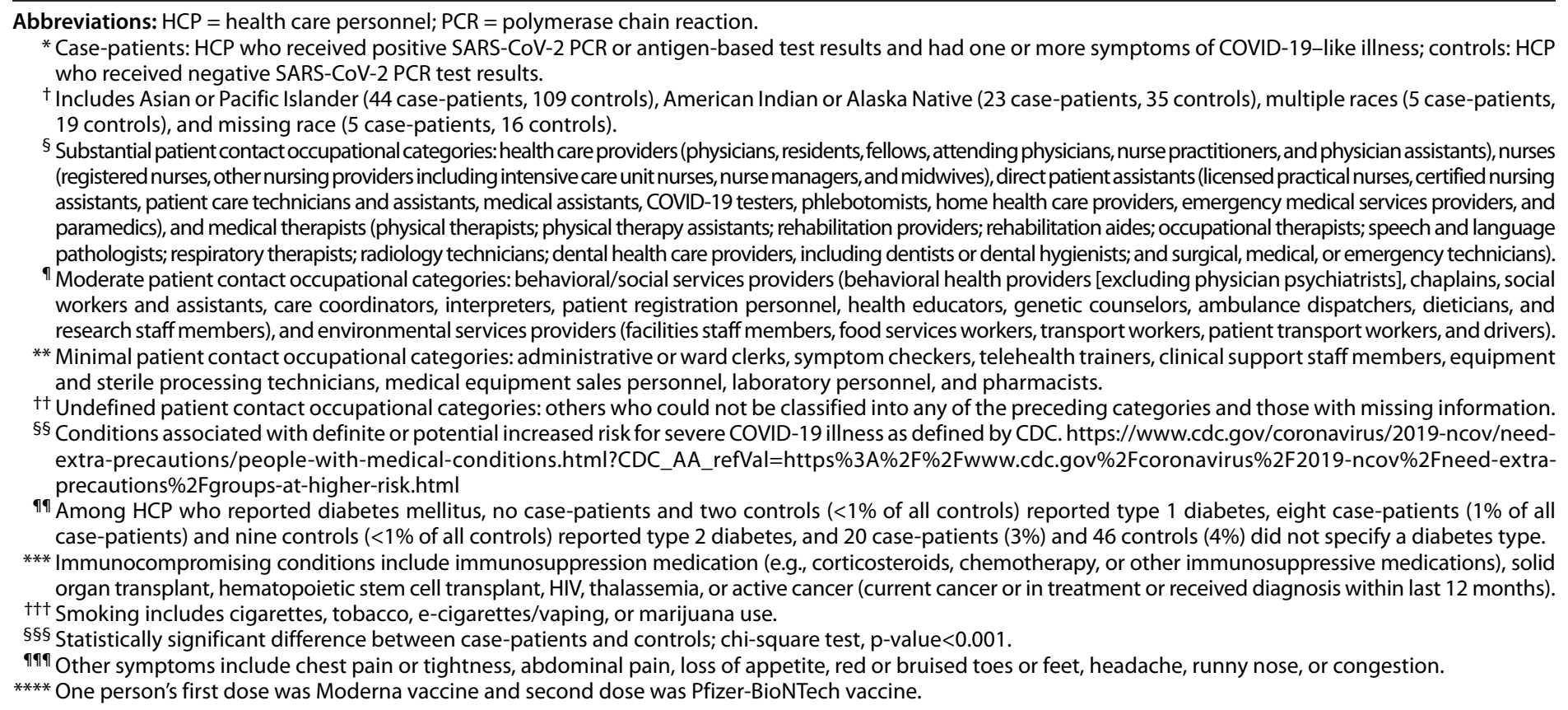

TABLE 2. COVID-19 vaccine effectiveness among health care personnel case-patients and controls, by number of COVID-19 vaccine doses received before SARS-CoV-2 test date - 33 U.S. sites, January-March 2021

\begin{tabular}{|c|c|c|c|c|}
\hline \multirow[b]{2}{*}{$\begin{array}{l}\text { Interval from } \\
\text { dose to test date }\end{array}$} & \multicolumn{2}{|c|}{ No. (\%) } & \multicolumn{2}{|c|}{$\begin{array}{c}\text { Vaccine effectiveness }{ }^{\dagger} \\
\%(95 \% \mathrm{Cl})\end{array}$} \\
\hline & $\begin{array}{l}\text { Case- } \\
\text { patients* } \\
(\mathrm{N}=623)\end{array}$ & $\begin{array}{l}\text { Controls* } \\
(\mathrm{N}=1,220)\end{array}$ & Unadjusted & Adjusted $^{\S}$ \\
\hline $\begin{array}{l}\text { Dose } 1 \\
\geq 14 \text { days }\end{array}$ & $64(10)$ & $241(20)$ & & \\
\hline $\begin{array}{l}\text { Dose } 2 \\
\leq 2 \text { days } \\
3-6 \text { days }\end{array}$ & $\begin{array}{l}5(<1) \\
16(3)\end{array}$ & $\begin{array}{r}109(9) \\
85(7)\end{array}$ & $82.2(75.1-87.3)$ & $81.7(74.3-86.9)$ \\
\hline$\geq 7$ days & $19(3)$ & $184(15)$ & 93.4 (86.4-96.8) & $93.5(86.5-96.9)$ \\
\hline
\end{tabular}

Abbreviations: $\mathrm{Cl}=$ confidence interval; $\mathrm{HCP}=$ health care personnel; $\mathrm{mOR}=$ matched odds ratio; $\mathrm{OR}=$ odds ratio; $\mathrm{PCR}=$ polymerase chain reaction; $\mathrm{VE}=$ vaccine effectiveness.

* Case-patients: HCP who received positive SARS-CoV-2 PCR or antigen-based test results and had one or more symptoms of COVID-19-like illness; controls: HCP who received negative SARS-CoV-2 PCR test results.

+ VE (Pfizer-BioNTech and Moderna) was estimated using a conditional logistic regression model accounting for matching by site of enrollment and week of test date.

$\S$ OR used in conditional logistic regression model to calculate VE was adjusted for age, race, and presence of underlying conditions: $\mathrm{VE}=100 \% \times(1-\mathrm{mOR})$.

effectiveness estimates in this and other studies were based on a short follow-up, the duration of this level of protection from a single dose is unknown.

The findings in this report are subject to at least four limitations. First, testing for SARS-CoV-2 infection among HCP was based on occupational health practices at each facility, and no changes in routine testing practices were reported after vaccine introduction. If vaccinated $\mathrm{HCP}$ were less likely to obtain testing than unvaccinated
HCP, the VE might have been underestimated. Alternatively, if postvaccination reactions increased the likelihood that vaccinated HCP would seek testing, the VE might have been overestimated. However, the sensitivity analysis excluding the interval of $0-2$ days after receipt of dose 2 , the interval during which most postvaccination reactions would be expected to occur, did not significantly change effectiveness estimates. Second, because of the limited sample size, effectiveness by vaccine product, presence of underlying medical conditions, and disease severity could not be estimated. In addition, because of limited statistical power, effectiveness estimates could not be adjusted for other potential confounders, such as use of personal protective equipment, occupational categories, or workplace or community exposures. Third, the VE estimates might not be generalizable to the U.S. adult population because racial/ ethnic minority groups disproportionately affected by COVID-19 and who may have had higher exposure risks in the community were underrepresented in this population, and the overall HCP population was younger than the general U.S. adult population. However, the study's geographic coverage was broad, representing the population of U.S. HCP, and vaccination data were obtained from multiple sources. Finally, although HCP with a known past acute SARS-CoV-2 infection were excluded, those whose previous infection was unknown could not be excluded. Data collection for this study is ongoing and will allow effectiveness to be evaluated by vaccine type and among HCP subgroups.

These interim results demonstrate that complete vaccination with authorized mRNA COVID-19 vaccines is highly effective in preventing symptomatic COVID-19 among HCP, 


\section{Summary}

What is already known about this topic?

Health care personnel (HCP) are at high risk for COVID-19. The early distribution of two mRNA COVID-19 vaccines (PfizerBioNTech and Moderna) to HCP provided an opportunity to examine vaccine effectiveness in a real-world setting.

What is added by this report?

The first U.S. multisite test-negative design vaccine effectiveness study among HCP found a single dose of Pfizer-BioNTech or Moderna COVID-19 vaccines to be $82 \%$ effective against symptomatic COVID-19 and 2 doses to be $94 \%$ effective.

What are the implications for public health practice?

The mRNA vaccines are highly effective at preventing symptomatic COVID-19 among U.S. HCP. High vaccination coverage among HCP and the general population is critical to prevent COVID-19 in the United States.

supporting the results of phase III trials and additional accruing evidence in recent observational studies. Real-world VE data are critical to guiding evolving COVID-19 vaccine policy. In addition to adherence to recommended infection control and prevention practices, a critical component of controlling the U.S. COVID-19 pandemic and protecting HCP is ensuring high coverage with safe and effective COVID-19 vaccines.

\section{Acknowledgments}

The health care personnel and health care systems who agreed to participate in this study; Jasmine Varghese; Taniece Eure; Rebecca M. Alkis Ramirez; Gregory Blazek; Kavitha Pathmarajah; Kelli Wallace; Alysia Horcher; Allison Schuette; Karin Hoth; Amanda Higgins; Brianna M. DiFronzo; Karen Hopcia; Theresa M. Orechia; Alexander B. Hill; Gabrielle Donohoe; Lily R. Johnsky; Jordyn M. Fofi; Steven E. Miyawaki; Jenson J. Kaithamattam; Michelle Chung; Nikita A. Umale; Mohammad Adrian Hasdianda; Guruprasad Jambaulikar; Tala Teymour; Maria Davila; Suzette Fernandez; Joshua Tiao; Eva Gonzalez; Reynaldo Padilla; Cynthia Delgado; Madeleine Manahan; Melanie Potts; Jessica Kuo; Alyssa Fowlds; Zoe Speight; Laurie Kemble; Danielle Beckham; Lori Wilkerson; Geneatra Green; Rachel Marrs; Katherine Schneider; Cathy Fairfield; Fred Ullrich; Virginia Mangolds; Morgan Nelson; Abigail Lopes; Scott Pelletier; Gloria Essien; Rebekah Peacock; Alan Jones; Bhagyashri Navalkele; Savannah Vann; Andrea Williams; Brooke Park; Eugene Melvin; Joel Rodgers; Nivedita Patkar; Delissa Tidwell-Hand; Whitney Covington; Michael C. Kurz; Peter Poerzgen; Layla A. Anderson; Kyle A. Steinbock; Christine D. Crider; Jennifer Smith; Ethan Lindgren; Linda Frank; Deborah Godine; Christopher Czaja; Anastasia Edwards; Elisabeth Harrington; Catherine Emanuel; Melissa Kellogg; Paula Clogher; Vivian Leung; Maya Dennis; Anisa Linton; James Meek; Linda Niccolai; Sara Niesobecki; Gwen Oliver; Monica Farley; Melissa Tobin-D’angelo; Stepy Thomas; Amy Tunali; Ingrid Zambrano; Erica Hazra; Kelli Williams; Kara Goldstone; Meaghan Woody; Timothy Walsh; Shannon Ball; Tameka Browne; Bailey Evenson; Rebecca Perlmutter; Emilija Motivans; Gerit Wagner; Tobias Leuthner; Ashley Fell; Kathy Como-Sabetti; Richard
Danila; Ruth Lynfield; Leslie Baken; Dana Essex; Marla Sievers; Sarah Shrum Davis; Yadira Salazar-Sanchez; Lezah Brown; Kristina Flores; Caroline Habrun; Cathleen Concannon; Jane A. Woods; Christine Hurley; Monika Samper; Gabriela Escutia; Judith A. Guzman-Cottrill; William Schaffner; Kathryn Billings; Melinda Eady; Danielle Ndi; Carol Epling; Kristen Said; Michael Yarrington; Michael Smith; Becky Smith; Christopher Polage; Rachel Addison; Alicia Nelson; Katherine Foy; Shaun Truelove; Lori Neihaus; Jayme Hughes; Annie Voskertchian; Mary Carl Froilan; Christine Fukuda; Jinal Makhija; Lahari Thotapalli; Brian Orleans; Morgan Millar; Tavis Huber; Matthew Doane; Kristina Stratford; Jacob Crook; Candace Haroldsen; Ling Yan; Jessica N. Zhang; Olivia G. Arter; Grace Yuan; Candace R. Miller; David McDonald; Caroline A. O'Neil; Jahnavi Bongu; Aurora Pop-Vicas; Joe Mcbride; Hannah Schopp; Amanda Young; Joe Perzynski; Michelle Zimbric; Fauzia Osman; Linda Stevens; Kelly Mitchell; Timothy Thomas; Matthew Hirschfeld; Brian Lefferts; Joseph Klejka; Donna Galbreath; James Tiesinga; Michael Bruce; Leisha Nolen; Wendy Petersen; Amy Swango Wilson; Vicki Vermillion; Brianna Smith; Scott LaBrie; Lauren Gillott; Shannon Williams; Madilyn Short; Christine Desnoyers; Lucinda Alexie; Evelyn Smith; Rachelle White; Marissa Friday.

\section{Vaccine Effectiveness Among Healthcare Personnel Study Team}

Anna Yousaf, CDC COVID-19 Response Team; Yunmi Chung, CDC COVID-19 Response Team; Jennifer Onukwube, CDC COVID-19 Response Team; Wei Xing, CDC COVID-19 Response Team; Bradley Clinansmith, University of Iowa, Iowa City, Iowa; Lisandra Uribe, Olive View-University of California Los Angeles Medical Center, Los Angeles, California; Kye E. Poronsky, Baystate Medical Center, Springfield, Massachusetts; Dean M. Hashimoto, Brigham and Women's Hospital, Boston, Massachusetts; Monica Bahamon, Jackson Memorial Hospital, Miami, Florida; Michelle St. Romain, Louisiana State University, New Orleans, Louisiana; Efrat Kean, Thomas Jefferson University Hospital, Philadelphia, Pennsylvania; Amy Stubbs, Truman Medical Center, Kansas City, Missouri; Sara Roy, University of Chicago, Chicago, Illinois; Gregory Volturo, University of Massachusetts, Worcester, Massachusetts; James Galbraith, University of Mississippi, Jackson, Mississippi; James C. Crosby, University of Alabama at Birmingham, Birmingham, Alabama; Megan R. Fuentes, University of Washington, Seattle, Washington; Mary Mulrow, Valleywise Health Medical Center, Phoenix, Arizona; Jane Lee, California Emerging Infections Program, Oakland, California; Helen Johnston, Colorado Department of Public Health and Environment, Denver, Colorado; AmberJean Hansen, Connecticut Emerging Infections Program, Yale School of Public Health, New Haven, Connecticut; Scott K. Fridkin, Georgia Emerging Infections Program, Atlanta, Georgia, and Emory University School of Medicine, Atlanta, Georgia; Lucy E. Wilson, Department of Emergency Health Services, University of Maryland, Baltimore County, Baltimore Maryland; Sara Lovett, Minnesota Emerging Infections Program, Minnesota Department of Health, St. Paul, Minnesota; Melissa Christian, University of New Mexico, Albuquerque, New Mexico, and New Mexico Emerging Infections Program, Santa $\mathrm{Fe}$, New Mexico; Christopher Myers, University of Rochester Medical Center/New York State-Rochester Emerging Infections Program, Rochester, New York; Valerie L.S. Ocampo, Public Health Division, 
Oregon Health Authority, Portland, Oregon; Keipp Talbot, Vanderbilt University Medical Center, Nashville, Tennessee; Jessica Seidelman, Duke Center for Antimicrobial Stewardship and Infection Prevention, Duke University School of Medicine, Durham, North Carolina; Aaron M. Milstone, Johns Hopkins School of Public Health, Baltimore, Maryland; Mary Hayden, Department of Medicine, Rush University, Medical Center, Chicago, Illinois; Matthew Samore, University of Utah, VA Salt Lake City Health Care System, Salt Lake City, Utah; Jennie H. Kwon, Washington University School of Medicine, St. Louis, Missouri; Daniel Shirley, University of Wisconsin-Madison, Madison, Wisconsin; Denise Dillard, Southcentral Foundation, Anchorage, Alaska; Jennifer Dobson, Yukon-Kuskokwim Health Corporation, Bethel, Alaska.

Corresponding author: Tamara Pilishvili, tdp4@cdc.gov.

${ }^{1}$ CDC COVID-19 Response Team; ${ }^{2}$ University of Iowa, Iowa City, Iowa; ${ }^{3}$ Olive View-University of California Los Angeles Medical Center, Los Angeles, California; ${ }^{4}$ Baystate Medical Center, Springfield, Massachusetts; ${ }^{5}$ Brigham and Women's Hospital, Boston, Massachusetts; 'Jackson Memorial Hospital, Miami, Florida; ${ }^{7}$ Louisiana State University, New Orleans, Louisiana; ${ }^{8}$ Thomas Jefferson University Hospital, Philadelphia, Pennsylvania; ${ }^{9}$ Truman Medical Center, Kansas City, Missouri; ${ }^{10}$ University of Chicago, Chicago, Illinois; ${ }^{11}$ University of Massachusetts, Worcester, Massachusetts; ${ }^{12}$ University of Mississippi, Jackson, Mississippi; ${ }^{13}$ University of Alabama at Birmingham, Birmingham, Alabama; ${ }^{14}$ University of California San Francisco, Fresno, California; ${ }^{15}$ University of Washington, Seattle, Washington; ${ }^{16}$ Valleywise Health Medical Center, Phoenix, Arizona; ${ }^{17}$ California Emerging Infections Program, Oakland, California; ${ }^{18}$ Colorado Department of Public Health and Environment, Denver, Colorado; ${ }^{19}$ Connecticut Emerging Infections Program, Yale School of Public Health, New Haven, Connecticut; ${ }^{20}$ Georgia Emerging Infections Program, Atlanta, Georgia;

${ }^{21}$ Emory University School of Medicine, Atlanta, Georgia; ${ }^{22}$ Maryland Department of Health, Baltimore, Maryland; ${ }^{23}$ Minnesota Emerging Infections Program, Minnesota Department of Health, St. Paul, Minnesota; ${ }^{24}$ University of New Mexico, Albuquerque, New Mexico; ${ }^{25} \mathrm{New}$ Mexico Emerging Infections Program, Santa Fe, New Mexico; ${ }^{26}$ University of Rochester Medical Center/New York State-Rochester Emerging Infections Program, Rochester, New York; 27Public Health Division Oregon Health Authority, Portland, Oregon; ${ }^{28}$ Vanderbilt University Medical Center, Nashville, Tennessee; ${ }^{29}$ Duke Center for Antimicrobial Stewardship and Infection Prevention, Duke University School of Medicine, Durham, North Carolina; ${ }^{30}$ Johns Hopkins School of Public Health, Baltimore, Maryland; ${ }^{31}$ Department of Medicine, Rush University Medical Center, Chicago, Illinois; ${ }^{32}$ University of Utah, VA Salt Lake City Health Care System, Salt Lake City, Utah; ${ }^{33}$ Washington University School of Medicine, St. Louis, Missouri; ${ }^{34}$ University of Wisconsin-Madison, Madison, Wisconsin;

${ }^{35}$ William S. Middleton Memorial Veterans Hospital, Madison, Wisconsin;

${ }^{36}$ Alaska Native Tribal Health Consortium, Anchorage, Alaska.
All authors have completed and submitted the International Committee of Medical Journal Editors form for disclosure of potential conflicts of interest. Deverick Anderson is the owner of Infection Control Education for Major Sports, LLC, and reports grants from the Agency for Healthcare Research and Quality and personal fees from UpToDate, outside the submitted work. Ghinwa Dumyati reports grants from Pfizer and personal fees from Roche Diagnostics. Gregory Moran reports grants from I-Mab Biopharma and BeiGene and personal fees from Light AI. Mark Steele reports personal fees from Light AI, during the conduct of the study. No other potential conflicts of interest were disclosed.

\section{References}

1. Hughes MM, Groenewold MR, Lessem SE, et al. Update: characteristics of health care personnel with COVID-19-United States, February 12-July 16, 2020. MMWR Morb Mortal Wkly Rep 2020;69:1364-8. PMID:32970661 https://doi.org/10.15585/mmwr. mm6938a3

2. Dooling K, Marin M, Wallace M, et al. The Advisory Committee on Immunization Practices' updated interim recommendation for allocation of COVID-19 vaccine-United States, December 2020. MMWR Morb Mortal Wkly Rep 2021;69:1657-60. PMID:33382671 https://doi. org/10.15585/mmwr.mm695152e2

3. Polack FP, Thomas SJ, Kitchin N, et al.; C4591001 Clinical Trial Group. Safety and efficacy of the BNT162b2 mRNA Covid-19 vaccine. N Engl J Med 2020;383:2603-15. PMID:33301246 https://doi.org/10.1056/ NEJMoa2034577

4. Baden LR, El Sahly HM, Essink B, et al.; COVE Study Group. Efficacy and safety of the mRNA-1273 SARS-CoV-2 vaccine. N Engl J Med 2021;384:403-16. PMID:33378609 https://doi.org/10.1056/ NEJMoa2035389

5. Dagan N, Barda N, Kepten E, et al. BNT162b2 mRNA Covid-19 vaccine in a nationwide mass vaccination setting. $\mathrm{N}$ Engl J Med 2021;384:1412-23. PMID:33626250 https://doi.org/10.1056/NEJMoa2101765

6. Thompson MG, Burgess JL, Naleway AL, et al. Interim estimates of vaccine effectiveness of BNT162b2 and mRNA-1273 COVID-19 vaccines in preventing SARS-CoV-2 infection among health care personnel, first responders, and other essential and frontline workerseight U.S. locations, December 2020-March 2021. MMWR Morb Mortal Wkly Rep 2021;70:495-500. PMID:33793460 https://doi.org/10.15585/ mmwr.mm7013e3

7. Hall VJ, Foulkes S, Saei A, et al.; SIREN Study Group. COVID-19 vaccine coverage in health-care workers in England and effectiveness of BNT162b2 mRNA vaccine against infection (SIREN): a prospective, multicentre, cohort study. Lancet 2021;397:1725-35. PMID:33901423 https://doi.org/10.1016/S0140-6736(21)00790-X 